\title{
New Error Measures to Evaluate Features on Three-Dimensional Scenes
}

\author{
Fabio Bellavia and Domenico Tegolo \\ Department of Mathematics and Computer Science, \\ University of Palermo, 90123, Palermo, Italy \\ \{fbellavia, domenico.tegolo\}@unipa.it
}

\begin{abstract}
In this paper new error measures to evaluate image features in 3D scenes are proposed and reviewed. The proposed error measures are designed to take into account feature shapes, and ground truth data can be easily estimated. As other approaches, they are not error-free and a quantitative evaluation is given according to the number of wrong matches and mismatches in order to assess their validity.
\end{abstract}

Keywords: Feature detector, feature descriptor, feature matching, feature comparison, overlap error, epipolar geometry.

\section{Introduction}

Feature-based computer vision applications have been widely used in the last decade [12. Their spread has increased the focus on feature detectors [8] and feature descriptors [7, as well as sparse matching algorithms [313. Besides, different evaluation strategies to assess their properties have been proposed in 871054.

The repeatability index introduced in [1] and the matching score [8] are common measures used for comparison. They have been adopted in well-known extensive comparisons for detectors 8, while precision-recall curves have been used for descriptors [7. Both the error measures described above have been applied to the Oxford dataset [9] which has become a standard de facto. The principal drawback of these approaches is to require a priori knowledge of all the possible correct matches between corresponding points in images. In the case of the planar scenes the Oxford dataset is made of, this can be trivially obtained by computing the planar homography from an exiguous number of hand-taken correspondences [6].

However, the use of features on 3D scenes is the most attractive and interesting topic for which nowadays new features are designed, so a relevant interest has risen in order to understand how they behave and their properties in a fully 3D environment. A strategy to overcome this issue was proposed in [5], where only two further image sequences, which contain fully $3 \mathrm{D}$ objects, are added to to extend the Oxford dataset. The trifocal tensor [6] is computed by an intermediate image and ground truth matches are recovered by using a dense matching 
strategy [12]. However, no information can be extracted for homogeneous regions or when occlusions are present, which can compromise the evaluation. Moreover, the complexity of the approach increases, which becomes less suitable for adding further image sequences to obtain a better evaluation.

A further evaluation of feature detectors and descriptors on 3D objects is reported in [10]. Differently from the other approaches, triplets of images are used leading to triplets of image correspondences instead of pairs. Correspondences are evaluated by epipolar geometry constrains [6] to build ROC curves used to analyse data and draw out conclusions. Though fundamental matrices [6] used to constrain triplets can be easily obtain by a relative low number of hand-take correspondences, the method is not completely error-free. A relevant number of correct matches can be discarded due to occlusions or by detector failures (about $50-70 \%$ ), while a few wrong matches can be incorrectly retained, especially when corresponding epipolar lines are near parallel (less than 10\%). Moreover this approach does not take into account the feature shapes, but it only considers the distance between the feature centres.

A last method described in [4] uses the pole-polar relationship to build an overlap error measure on segments lying on corresponding feature pairs. Only the fundamental matrix is required to compute the ground truth, but wrong matches can be accepted. According to the authors, this can happen with a low probability they did not experimentally measured.

In this paper new error measures to evaluate image features in 3D scenes are proposed and reviewed, also employing strategies which use image triplets as in [10]. The proposed measures are designed to take into account the feature shapes and, moreover, ground truth data can be easily estimated for 3D scenes. As other approaches, they are not error-free and in order to assess their validity, a quantitative evaluation is given according to the number of wrong matches and mismatches. Moreover, the method described in [4] has been included. The resulting quantitative analysis also provides clues on the possible number of wrong matches retained when these measures are employed to validate matching algorithms. In Sect. 2, the proposed error measures are introduced, while in Sect. 3] the experimental setup to assess their validity and the results are described. Finally, in Sect. 4 conclusions and future works are discussed.

\section{The New Error Measures}

\subsection{Definition}

Given a stereo image pair $\left(I_{1}, I_{2}\right)$ and corresponding points $\mathbf{x}_{1} \in I_{1}, \mathbf{x}_{2} \in I_{2}$ in homogeneous coordinates, the fundamental matrix $\mathrm{F}$ determines the relation $\mathbf{x}_{2}^{\mathrm{T}} \mathbf{F} \mathbf{x}_{1}=0$. Geometrically the point $\mathbf{x}_{1}$ is constrained to lie on the epipolar line $\mathbf{l}_{1}=\mathbf{x}_{2}^{\mathrm{T}} \mathrm{F}$, and in similar way $\mathbf{x}_{2}$ on $\mathbf{l}_{2}=\mathbf{x}_{1}^{\mathrm{T}} \mathrm{F}^{\mathrm{T}}$ in the corresponding image. Epipolar lines pass through the epipoles $\mathbf{e}_{1} \in I_{1}, \mathbf{e}_{2} \in I_{2}$, which are respectively the right, left null-space of $\mathrm{F}$. The ground truth fundamental matrix F can be extracted by an exiguous number of hand-taken correspondences by using a method described in [6]. 
Let $\mathcal{R}_{1} \in I_{1}, \mathcal{R}_{2} \in I_{2}$ be two feature patches centred in $\mathbf{x}_{1}, \mathbf{x}_{2}$. Their shapes are elliptical disks, as commonly defined by feature detectors, with minor and major axes respectively $\alpha_{\text {min }_{i}}, \alpha_{\max _{i}}, i \in\{1,2\}$. Let also $d(\cdot, \cdot)$ define the Euclidean distance between points or between a point and a line according to its arguments. The first error measure in the image $I_{i}, i \in\{1,2\}$, for the a feature pair $\left(\mathcal{R}_{1}, \mathcal{R}_{2}\right)$ is defined as

$$
\xi_{i}=\min \left(\frac{d\left(\mathbf{x}_{i}, \mathbf{l}_{i}\right)}{\alpha_{\min _{i}}}, 1\right)
$$

that is, the epipolar distance between the feature centre and its epipolar line computed by using the corresponding point in the other image is normalized by the minor axis of the feature ellipse (see Fig. 1(a)). The error $\xi$ on both the images is

$$
\xi=\max _{i} \xi_{i} \quad i \in\{1,2\}
$$

In similar way the error $\kappa_{i}$ in the image $I_{i}$ is defined as

$$
\kappa_{i}=\min \left(\frac{d\left(\mathbf{x}_{i}, \mathbf{l}_{i}\right)}{2 d\left(\mathbf{x}_{i}, \mathbf{p}_{i}\right)}, 1\right)
$$

where $\mathbf{p}_{i}$ is the intersection between the feature elliptical boundary and the line perpendicular to the epipolar line $\mathbf{l}_{i}$ through $\mathbf{x}_{i}$ (see Fig. 1(b)). Analogously, the error $\kappa$ on both the image is

$$
\kappa=\max _{i} \kappa_{i} \quad i \in\{1,2\}
$$

Both the error measures $\xi$ and $\kappa$ take into account the shape of the feature patch, but the former, by considering only the maximum circle inside the feature ellipse, makes a more pessimistic assumption about the correctness of the features as extracted by the feature detector (see Fig. 1 (a-b)). The error for both $\xi$ and $\kappa$ achieves the maximal value of 1 roughly when the reprojected feature ellipse would not touch the considered feature ellipse (see Fig. 1(a-b)). Moreover, the maximal error between the two images is retained instead of their average, as it is the symmetric error, because the former solution is more constraining.

The last error measure extends that introduced in [4] which will be described for clarity. The tangency relation is preserved by perspective projection, which retains incidence relations. Let $\mathbf{l}_{t_{1}^{i}}, \mathbf{l}_{t_{2}^{i}}$ be the epipolar lines in the image $I_{i}$, $i \in\{1,2\}$ corresponding respectively to the tangent points $\mathbf{t}_{1}^{\bar{i}}, \mathbf{t}_{2}^{\bar{i}}$ in $I_{\bar{i}}$ through the epipole $\mathbf{e}_{\bar{i}}$ to the feature ellipse $\mathcal{R}_{\bar{i}}$, where $\bar{i}=3-i$ (i.e. $I_{i}$ and $I_{\bar{i}}$ are the complementary images of the stereo pair). Let $\mathbf{q}_{i}$ be the line through the tangent points $\mathbf{t}_{1}^{i}, \mathbf{t}_{2}^{i}$. The intersection points $\mathbf{r}_{1}^{i}, \mathbf{r}_{2}^{i}$ of $\mathbf{l}_{t_{1}^{i}}, \mathbf{l}_{t_{2}^{i}}$ with the line $\mathbf{q}_{i}$ are used to define a linear overlap error $\varepsilon_{l_{i}}$ in the image $I_{i}$ (see Fig. 1(c)) as follows

$$
\varepsilon_{l_{i}}=1-\frac{\max \left(0, \min \left(t_{h}^{i}, r_{h}^{i}\right)-\max \left(t_{l}^{i}, r_{l}^{i}\right)\right)}{\max \left(t_{h}^{i}, r_{h}^{i}\right)-\min \left(t_{l}^{i}, r_{l}^{i}\right)}
$$

where $r_{h}^{i}$ and $r_{l}^{i}$ are the higher and lower linear coordinates of $\mathbf{r}_{1}^{i}$ and $\mathbf{r}_{2}^{i}$ on the line $\mathbf{q}_{i}$ respectively, according to a defined direction, and in similar way $t_{h}^{i}$ and 
$t_{l}^{i}$ for $\mathbf{t}_{1}^{i}$ and $\mathbf{t}_{2}^{i}$ respectively $\left(r_{h}^{i} \equiv \mathbf{r}_{1}^{i}, t_{h}^{i} \equiv \mathbf{t}_{1}^{i}, r_{l}^{i} \equiv \mathbf{r}_{2}^{i}, t_{h}^{i} \equiv \mathbf{t}_{2}^{i}\right.$ in the example of Fig. 1(c)). The final error on both the images is defined as the average error

$$
\varepsilon_{l_{\text {avg }}}=\frac{\varepsilon_{1}+\varepsilon_{2}}{2}
$$

In particular the authors consider a match correct if $\varepsilon_{l_{a v g}}<0.2$ [4]. In the next, for the same motivation described above the follow definition of linear overlap error $\varepsilon_{l}$ will be used instead

$$
\varepsilon_{l}=\max _{i} \varepsilon_{l_{i}} \quad i \in\{1,2\}
$$

An extension to the linear overlap error measure $\varepsilon_{l}$ is proposed, by observing that not only the correspondence between epipoles is available, but also fixed correspondences $\left(\mathbf{w}_{1}^{k}, \mathbf{w}_{2}^{k}\right)$, with $k=\{1, \ldots, m\}$ and $\mathbf{w}_{i}^{k} \in I_{i}$, provided by further hand-taken points used to compute the fundamental matrix. By combining their tangent lines to the feature ellipse it is possible to obtain an inscribed and a circumscribed quadrilaterals, which can be used to approximate the feature ellipse (see Fig. 1(d)). Both the quadrilaterals can be approximately projected through the fundamental matrix, thus an approximate overlap error $\varepsilon_{q}$ between the feature patches can be computed, similar to the standard definition of overlap error $\varepsilon$ between surface patches used in [8]

$$
\varepsilon\left(\mathcal{R}_{1}, \mathcal{R}_{2}\right)=1-\frac{\mathcal{R}_{1} \cap \mathcal{R}_{2}}{\mathcal{R}_{1} \cup \mathcal{R}_{2}}
$$

In detail, for two pairs of fixed corresponding points $\left(\mathbf{w}_{1}^{k}, \mathbf{w}_{2}^{k}\right)$ and $\left(\mathbf{w}_{1}^{s}, \mathbf{w}_{2}^{s}\right)$, $k, s \in\{1, \ldots, m\}, k \neq s$, on the image $I_{i}$, one can obtain the quadrilateral $\mathcal{Q}_{i}$ circumscribed to the feature ellipse $\mathcal{R}_{i}$ by intersecting the tangent lines to $\mathcal{R}_{i}$ through the fixed points $\mathbf{w}_{i}^{k}, \mathbf{w}_{i}^{s}$ (see Fig. 1(d)). The corresponding tangent points are used instead to get the quadrilateral $\mathcal{Q}_{i}^{\star}$ inscribed to the ellipse $\mathcal{R}_{i}$ (see Fig. 1(d)). With an abuse of notation the area of the ellipse $\mathcal{R}_{i}$ can be roughly approximated by the average area between the two quadrilaterals $\mathcal{Q}_{i}$ and $\mathcal{Q}_{i}^{\star}$

$$
\mathcal{R}_{i} \approx \frac{\mathcal{Q}_{i}+\mathcal{Q}_{i}^{\star}}{2}
$$

Reprojected tangent points $\mathbf{v}_{i}^{z}, z \in\{1, \ldots, 4\}$ from the feature $\mathcal{R}_{\bar{i}}$ of the complementary image $I_{\bar{i}}$ can be approximated as done for $\mathbf{r}_{1}^{i}, \mathbf{r}_{2}^{i}$ in Equ. 5. The epipole is substituted in turn with the fixed points $\mathbf{w}_{i} \frac{k}{i}$ in the definition of the linear overlap error $\varepsilon_{l_{\text {avg }}}$. An approximate reprojection $\mathcal{P}_{i}^{\star}$ in $I_{i}$ of the inscribed quadrilateral $\mathcal{Q}_{i}^{\star}$ is obtained by connecting the points $\mathbf{v}_{i}^{z}$ (see Fig. 11(d)). The intersection points of lines through $\mathbf{v}_{i}^{z}$ and the corresponding fixed points $\mathbf{w}_{i}^{k}, \mathbf{w}_{i}^{s}$ form instead an approximate reprojection $\mathcal{P}_{i}$ of the circumscribed quadrilateral $\mathcal{Q}_{\bar{i}}$ (see Fig. $1(\mathrm{~d})$ ).

By taking into account the formula to evaluate the approximated area (see Equ. 9), a further approximated overlap error $\varepsilon_{q_{i}}$ in the image $I_{i}$ is defined as

$$
\varepsilon_{q_{i}}=\frac{\varepsilon\left(\mathcal{Q}_{i}, \mathcal{P}_{i}\right)+\varepsilon\left(\mathcal{Q}_{i}^{\star}, \mathcal{P}_{i}^{\star}\right)}{2}
$$


and the corresponding approximate overlap error $\varepsilon_{q}$ on both the image is

$$
\varepsilon_{q}=\max _{i} \varepsilon_{q_{i}} \quad i \in\{1,2\}
$$

The approximated overlap error $\varepsilon_{q}$ strictly depends on the choice of the two corresponding matches with indexes $k, s$, and the computation can suffer of numerical instability because the projected tangent points $\mathbf{v}_{i}^{z}$ are derived by using epipolar lines which are close together and almost parallel, especially when the epipoles are far away from the image centres. This issue can be alleviated empirically by using only index pairs $(k, s)$ for which the diagonals of the quadrilaterals $\mathcal{Q}_{i}, \mathcal{Q}_{i}^{\star}, \mathcal{P}_{i}, \mathcal{P}_{i}^{\star}$ form a minimum angle of at least $\pi / 3$. Moreover, in order to obtain tangent lines which are not almost parallel, the fixed points $\mathbf{w}_{i}^{k}, \mathbf{w}_{i}^{s}$ are considered if they are three times the semi-major axis of the feature ellipse $\mathcal{R}_{i}$ far away from the centre $\mathbf{x}_{i}$. Finally, for each feature pair $\left(\mathcal{R}_{1}, \mathcal{R}_{2}\right)$ the minimum approximated overlap error $\varepsilon_{q}$ among all the admissible pairs of indexes $(k, s)$ is retained.

\subsection{Extension to Image Triplets}

All the described error measures $\xi, \kappa, \varepsilon_{l}$ and $\varepsilon_{q}$ depend on the epipolar constrain, thus they cannot characterize wrong matches when they lie close to the epipolar line of the true corresponding image feature. This however does not happen frequently, as it has been observed in the experimental valuation.

In order to alleviate this issue, three strategies similar to that proposed in [10] have been considered, which make use of triplet of images $I_{i}, i \in\{1,2,3\}$. Let $\left(I_{1}, I_{2}\right),\left(I_{1}, I_{3}\right),\left(I_{2}, I_{3}\right)$ be the three stereo pairs, and $\gamma \in\left\{\xi, \kappa, \varepsilon_{l}, \varepsilon_{q}\right\}$ an error measure. Consistent chains of features between the stereo pairs are defined by the chain error $\gamma_{\delta_{t}}$, according to different strategies $\delta_{t}, t \in\{1,2,3\}$. This error is associated back to features pair, so that feature chains and pairs are accepted if their respective error is $\gamma_{\delta_{t}}<1$.

The first strategy $\delta_{1}$ acts as follows. If $\left(\mathcal{R}_{k_{1}}, \mathcal{R}_{k_{2}}, \mathcal{R}_{k_{3}}\right)$ is the $k$-th triplet among all the possible triplets of feature points, with $\mathcal{R}_{k_{i}} \in I_{i}$, let $\gamma\left(z_{i}, z_{j}\right)$ be the error corresponding to the $z$-th feature pair $\left(\mathcal{R}_{z_{i}}, \mathcal{R}_{z_{j}}\right)$ belonging to the stereo pair $\left(I_{i}, I_{j}\right), i, j=\{1,2,3\}$. Clearly, $\gamma\left(z_{i}, z_{j}\right)=\gamma\left(z_{j}, z_{i}\right)$ and $\gamma\left(z_{i}, z_{i}\right)=$ $\gamma\left(z_{j}, z_{j}\right)=0$. The maximum among all the errors of the feature pairs inside the triplet, is associated back to the triplet

$$
\gamma_{\delta_{1}}\left(\mathcal{R}_{k_{1}}, \mathcal{R}_{k_{2}}, \mathcal{R}_{k_{3}}\right)=\max _{z_{i}, z_{j} \in\left\{k_{1}, k_{2}, k_{3}\right\}} \gamma\left(\mathcal{R}_{z_{i}}, \mathcal{R}_{z_{j}}\right)
$$

The triplet forms a consistent virtual chain if $\gamma_{\delta_{1}}<1$. A pair of features is retained if it belongs to a consistent virtual chain. That is, the relation $\gamma_{\delta_{1}}<1$ holds for the pair, defined as

$$
\gamma_{\delta_{1}}\left(\mathcal{R}_{z_{i}}, \mathcal{R}_{z_{j}}\right)=\min _{z_{i}, z_{j} \in\left\{k_{1}, k_{2}, k_{3}\right\}} \gamma_{\delta_{1}}\left(\mathcal{R}_{k_{1}}, \mathcal{R}_{k_{2}}, \mathcal{R}_{k_{3}}\right)
$$

In similar way two further match selection strategies $\delta_{2}, \delta_{3}$ are defined, but clues about the correctness of the matches provided by the feature detector and 
descriptor are used instead to provide more insight. In details, let $\left[\mathcal{R}_{k_{i}}, \mathcal{R}_{k_{j}}\right]$ be the $k$-th match between two features to be evaluated in the stereo pair $\left(I_{i}, I_{j}\right)$ (e.g. the features have been ranked according to the feature descriptor similarity and a threshold was applied to retain the putative correct ones). Define the error associated to a pair of matches, which form a partial chain, as

$$
\gamma_{\delta_{2}}\left(\left[\mathcal{R}_{k_{i}}, \mathcal{R}_{k_{j}}\right],\left[\mathcal{R}_{\bar{k}_{j}}, \mathcal{R}_{\bar{k}_{w}}\right]\right)=\max _{z_{p}, z_{q} \in\left\{k_{i}, k_{j}, \bar{k}_{j}, \bar{k}_{w}\right\}} \gamma\left(\mathcal{R}_{z_{p}}, \mathcal{R}_{z_{q}}\right)
$$

To be noted that virtual matches are considered, e.g. the pair $\left(\mathcal{R}_{k_{i}}, \mathcal{R}_{\bar{k}_{j}}\right)$. A partial chain is consistent if $\gamma_{\delta_{2}}<1$. A generic pair $\left(\mathcal{R}_{z_{p}}, \mathcal{R}_{z_{q}}\right)$ is retained if it belongs to a consistent partial chain, that is $\gamma_{\delta_{2}}<1$ for the pair, where

$$
\gamma_{\delta_{2}}\left(\mathcal{R}_{z_{p}}, \mathcal{R}_{z_{q}}\right)=\min _{z_{p}, z_{q} \in\left\{k_{i}, k_{j}, \bar{k}_{j}, \bar{k}_{w}\right\}} \gamma_{\delta_{2}}\left(\left[\mathcal{R}_{k_{i}}, \mathcal{R}_{k_{j}}\right],\left[\mathcal{R}_{\bar{k}_{j}}, \mathcal{R}_{\bar{k}_{w}}\right]\right)
$$

In the last strategy $\gamma_{\delta_{3}}$ triplets of matches are used instead of pairs to form a full chain of matches, i.e. triplets of the form

$$
\left(\left[\mathcal{R}_{k_{i}}, \mathcal{R}_{k_{j}}\right],\left[\mathcal{R}_{\bar{k}_{j}}, \mathcal{R}_{\bar{k}_{w}}\right],\left[\mathcal{R}_{\underline{k}_{w}}, \mathcal{R}_{\underline{k}_{i}}\right]\right)
$$

The relation $\gamma_{\delta_{3}}$ for both triplets of matches and pair of features is defined analogously.

The proposed strategies $\delta_{i}$ are only used to remove putative wrong matches, they are not used as actual error measures, because they provide very high error values. In particular only matches belonging to consistent chains, i.e $\gamma_{\delta_{i}}<1$, are retained and scored according to $\gamma$. Though these strategies can increase the quality of the matches classification, it should be noted that when large portions of the images are affected by occlusions, a relevant fraction of good matches can be discarded because consistent chain cannot be formed.

\section{Measure Assessment}

\subsection{Experimental Setup}

In order to compare the robustness of proposed error measures $\gamma \in\left\{\xi, \kappa, \varepsilon_{l}, \varepsilon_{q}\right\}$ a set of 10 sequences, consisting of three images of a 3D scene taken from different points of view, have been used, for a total of 30 stereo image pairs. Different degrees of image transformations are present and their final effect, which depends on different degrees of occlusion, baseline distances and camera orientations, is not quantitatively computable. However the average behaviour of the proposed error measures could be deduced.

The Sampson and the epipolar error measures are not included in this evaluation because they cannot provide an error estimation relative to the feature shape. However, while $\xi$ and $\kappa$ are normalized forms of the epipolar error, in the case of the Sampson error a straight normalization cannot be deduced. 
The ground truth fundamental matrix for each stereo pair was computed using the normalized eight-point algorithms [6] on hand-taken correspondences. Three different users provided each more than 50 homogeneously distributed correspondences for every stereo pair, which have been merged together in order to get a more stable fundamental matrix.

To estimate a ground truth in order to assess the goodness of the error measures $\gamma$ and of the selection strategies $\delta_{i}, i \in\{1,2,3\}$, features extracted by the HarrisZ detector [2] have been ranked using the sGLOH descriptor [1] and matches have been supervised by an user, so that if corresponding features in a match share a minimal region then the match is considered correct. According to this match classification and by taking into account the error measure definitions, every match with $\gamma<1$ should be classified as correct.

The HarrisZ detector is a corner-based feature detector while the sGLOH descriptor is based on gradient histograms, they both have proven to be robust and stable 21. To be noted that misclassified matches should depend on the inherent structure of stereo pair, not on the detector and the descriptor used in the assessment. In order to validate this statement, the use of further feature detectors and descriptors is planned.

Precision-recall curves have been computed for each stereo pairs. The recall is defined as the fraction of correct matches discarded by $\gamma<1$, while the precision is the fraction of correct classified matches. In order to draw the curve, matches have been ranked according to increasing error values. A marker underlines an error increase by a step of 0.1 for each curve. To be observed that not only the precision is critical to evaluate features detectors and descriptors as underlined in [10, but also the recall should be taken into consideration.

Moreover, while the denominator in the recall ratio is fixed to the number of ground truth correct matches, the denominator of the precision ratio varies as the error value $\gamma$ increases. To be noted that the recall monotonically increases as the error threshold increases while this does not hold for the precision which varies in $[0,1]$. This can give rise to quasi-horizontal oscillations in the precision-recall curves, mostly noticeable for low values of the error threshold, which provide an indicator of the stability of the error measures.

The plots have been computed for the different errors $\gamma$, along with selection strategies $\delta_{i}$. Any combination $\varepsilon_{q}, \delta_{i}$ was omitted, because it requires to compute the error value $\varepsilon_{q}$ for each feature pair combination, not only for matched pairs. This is not feasible due to the huge computational time required.

\subsection{Results}

Plots are shown in Fig. 2. more detailed results can be found online 1 due to lack of space. A summarizing plot for each error measure on the whole image sequences is not shown, since it would be misleading due to the different and not comparable transformations it should average. According to the plots, all the proposed error measures reach a precision of about $80-95 \%$ for a recall around

\footnotetext{
${ }^{1}$ http://www.math.unipa.it/ fbellavia/plots.zip
} 
85-95\% on average, from where the curve is stabilized and slowly increases the recall, but also decreases the precision. This point is achieved for error values $\xi, \kappa, \varepsilon_{l} \approx 0.5$, and $\varepsilon_{q} \approx 0.9$ respectively, which means that the surface error $\varepsilon_{q}$ better approximates the overlap error $\varepsilon$, while for the other linear errors $\xi, \kappa, \varepsilon_{l}$ normalization to semi-axes instead of axes is a better choice.

It can also be noted that the plots for the error measures $\varepsilon_{q}, \varepsilon_{l}$ slighty increase faster than $\xi, \kappa$. Only for the kermit sequence (see Fig. 2, middle row) a very low precision is achieved, however it happens for a high point view change (about $\pi / 2)$. Moreover, it seems that $\varepsilon_{l}$ measure degrades when the epipoles are inside the images, as it can be noted in the corridor sequence (see Fig. 2, top row), while this does not occur for the analogous error $\varepsilon_{q}$.

About the application of the different selection strategies $\delta_{i}$, the error measures $\xi, \kappa$ take more benefits with respect to $\varepsilon_{l}$ in terms of faster curve increment, but also in terms of stability. The threshold at 0.5 for the error measures $\xi, \kappa$, $\varepsilon_{l}$ is no more requested in order to achieve good precision values, which means that the filtering is effective. As more constrains are added increasing the index $i$, better precision is achieved. However the decrease of the recall is usually greater and can reach about $50 \%$ for $i=3$ in the experiments. According to these observations, the partial chain strategy $\delta_{2}$ seems to provide a reasonable compromise.

As further remarks, the two measures $\xi, \kappa$ are very similar in results, so $\xi$ should be preferred because faster. Also $\varepsilon_{l}$ and $\varepsilon_{q}$ obtain similar results, however while the former is faster, the latter can better handle some epipolar stereo configurations and it seems to provide better values in terms of overlap error approximation. Both $\xi, \kappa$ are faster than $\varepsilon_{l}, \varepsilon_{q}$, however the last two error measures provide better results in terms of curve increase.

Though the precision can be relatively low for accurate comparison, qualitative evaluation can be drawn out using the proposed error measures. Moreover it must be remembered that in the experiments a match is correct if a minimum overlap is present, which means that in common test requirements, i.e. an overlap error $\varepsilon \approx 0.5$, the precision is higher with a good recall.

\section{Conclusion and Future Works}

In this paper new error measures for evaluation of features on 3D scenes have been presented. They make use of the feature shape and ground truth data can be easily estimated. Their robustness have been evaluated according to precisionrecall curves, which have shown their effectiveness. Though not error-free, qualitative evaluations can be carried out according to them. Moreover, the analysis of the plots of the proposed error measures can be used as a flexible guideline for their application, which mainly depends on the particular evaluation task.

While the linear overlap approximation $\varepsilon_{l}$ presented in [4] and its proposed extension to surface $\varepsilon_{q}$ provide relatively better approximation of the overlap error $\varepsilon$, the normalized version of the epipolar error $\kappa$ and $\xi$ are faster, in particular $\xi$. Furthermore an increase in precision can be obtained by using triplets, 
however with a lost in recall. The strategy $\delta_{2}$, which makes use of partial chain of features, seems a good compromise.

Future works will include further experiments using other feature detectors and descriptors to assess the results, as well as the use of finer match ground truth, i.e. instead of consider matches correct if they have a minimal overlap $\varepsilon<1$, different overlap error could be applied. Moreover, the error measure $\xi$ is very fast and could be applied to retrieve inliers in a RANSAC framework, as it done by MSAC [13], but by taking into account also the feature shape.

Acknowledgments. Thanks to Cesare Valenti, for the useful discussions and for his help in the ground truth estimation of the fundamental matrices.

This work has also been partially supported by a grant "Fondo per il Potenziamento della Ricerca del Dipartimento di Matematica e Applicazioni dellUniversità degli Studi di Palermo".

\section{References}

1. Bellavia, F., Tegolo, D., Trucco, E.: Improving SIFT-based descriptors stability to rotations. In: International Conference on Pattern Recognition (2010)

2. Bellavia, F., Tegolo, D., Valenti, C.: Improving Harris corner selection strategy. IET Computer Vision 5(2) (2011)

3. Fischler, M.A., Bolles, R.C.: Random sample consensus: a paradigm for model fitting with applications to image analysis and automated cartography. Communications of the ACM 24(6), 381-395 (1981)

4. Forssén, P., Lowe, D.G.: Shape descriptors for maximally stable extremal regions. In: International Conference on Computer Vision. IEEE Computer Society, Los Alamitos (2007)

5. Fraundorfer, F., Bischof, H.: A novel performance evaluation method of local detectors on non-planar scenes. In: IEEE Conference on Computer Vision and Pattern Recognition, p. 33. IEEE Computer Society, Los Alamitos (2005)

6. Hartley, R., Zisserman, A.: Multiple View Geometry in Computer Vision. Cambridge University Press, Cambridge (2000)

7. Mikolajczyk, K., Schmid, C.: A performance evaluation of local descriptors. IEEE Transactions on Pattern Analysis and Machine Intelligence 27(10), 1615-1630 (2005)

8. Mikolajczyk, K., Tuytelaars, T., Schmid, C., Zisserman, A., Matas, J., Schaffalitzky, F., Kadir, T., Van Gool, L.: A comparison of affine region detectors. International Journal of Computer Vision 65(1-2), 43-72 (2005)

9. Mikolajczyk, K., Tuytelaars, T., et al.: Affine covariant features (2010), http://www.robots.ox.ac.uk/ vgg/research/affine

10. Moreels, P., Perona, P.: Evaluation of features detectors and descriptors based on 3d objects. International Journal of Computer Vision 73(3), 263-284 (2007)

11. Schmid, C., Mohr, R., Bauckhage, C.: Evaluation of interest point detectors. International Journal of Computer Vision 37(2), 151-172 (2000)

12. Szeliski, R.: Computer Vision: Algorithms and Applications. Springer, Heidelberg (2010)

13. Torr, P.H.S., Zisserman, A.: Robust computation and parametrization of multiple view relations. In: International Conference on Computer Vision, p. 727. IEEE Computer Society, Los Alamitos (1998) 


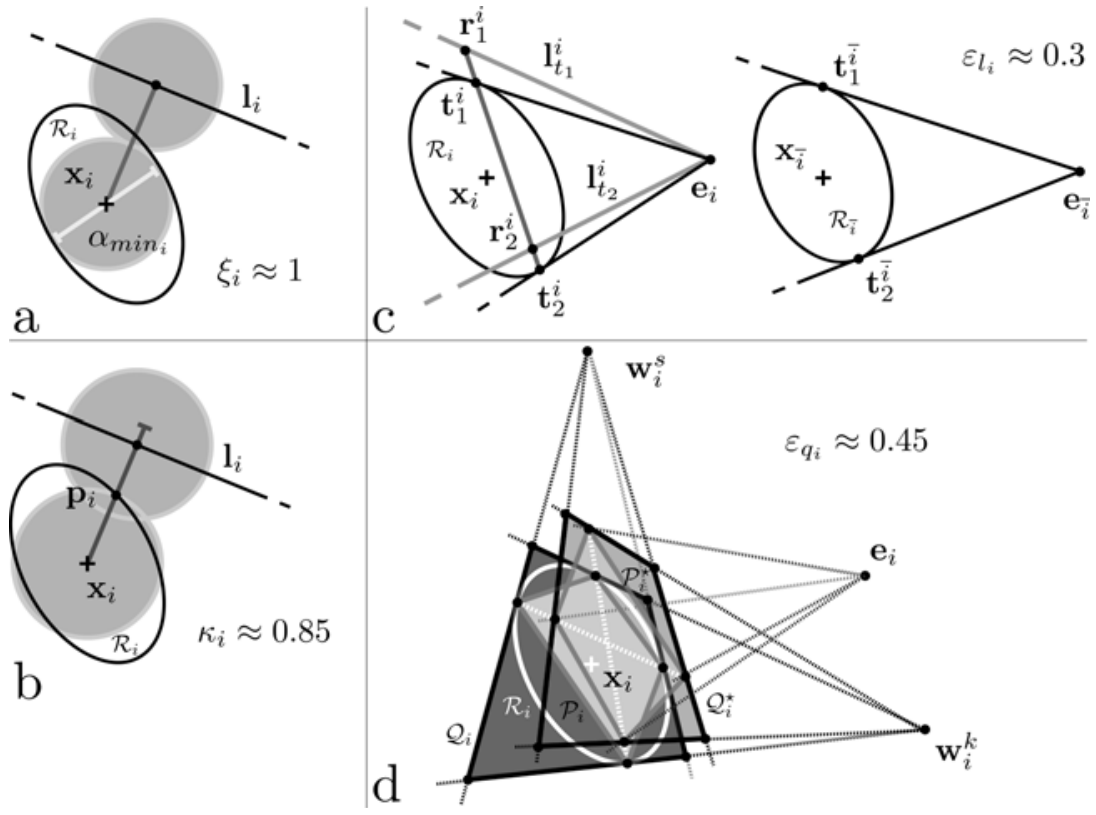

Fig. 1. Error measures $\xi(\mathrm{a}), \kappa(\mathrm{b}), \varepsilon_{l}(\mathrm{c})$ and $\varepsilon_{q}(\mathrm{~d})$
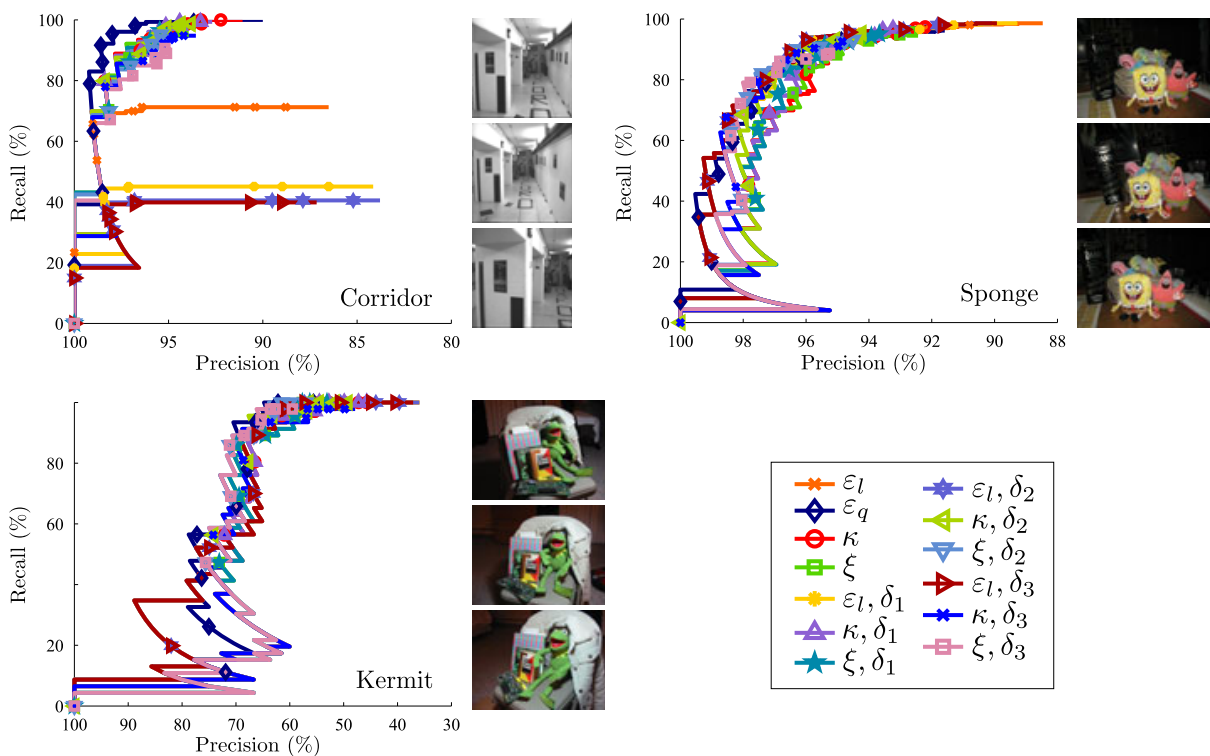

Fig. 2. Precision-recall curves (left) for some image sequences (right). The intermediate image to compute the strategies $\delta_{i}$ is in the corresponding middle row. 